\title{
Analysis of Rectangular Kirchhoff Plate on Winkler Foundation using Finite Fourier Sine Transform Method
}

\author{
Mama B.O. ${ }^{1}$, Ike C.C. ${ }^{* 2}$, Onah H.N. ${ }^{3}$, Nwoji C.U. ${ }^{4}$ \\ ${ }^{I}$ Dept of Civil Engineering University of Nigeria, Nsukka, Enugu State, Nigeria. \\ ${ }^{2}$ Dept of Civil Engineering Enugu State University of Science \& Technology, Enugu State, Nigeria. \\ ${ }^{3}$ Dept of Civil Engineering University of Nigeria, Nsukka, Enugu State, Nigeria. \\ ${ }^{4}$ Dept of Civil Engineering University of Nigeria, Nsukka, Enugu State, Nigeria.
}

\begin{abstract}
In this work, the rectangular Kirchhoff plate on Winkler foundation problem was solved using the finite Fourier Sine transform method. The plate considered was assumed homogeneous and isotropic and simply supported at the four edges $x=0, x=a, y=0$ and $y=b$. Application of the double finite Fourier sine transformation to the governing fourth order partial differential equation simplified the boundary value problem to an algebraic equation in terms of the deflection in the transform space variables, $m, n$. The deflection obtained in the transform space variables was inverted to obtain the solution in the problem domain variables Particular cases of transverse distributed load namely point load at any point on the plate and uniform load on the entire plate were considered and solved. The solutions were found to be identical with the exact solutions in literature obtained using the Navier series method.
\end{abstract}

Keywords: double finite Fourier sine transform method, Kirchhoff plate, Winkler foundations.

\section{Introduction}

A transversely loaded plate resting on an elastic foundation may be used to model the behaviour of reinforced concrete road pavement, an airport runway, or a mat (raft foundation) [1]. There also exist many other problems of considerable practical and engineering significance that are described with partial differential equations that are similar in form to the equations of plates on elastic foundations. For instance, the governing equation describing a shallow shell is analogous to the equation of a plate on an elastic foundation [2]. The principal difficulty of the plate on elastic foundation problem is in the mathematical idealization of the elastic foundation. Several mathematical descriptions of the elastic foundation are found in the literature $[3,4,5,6,7$, 8, 9]. Some are Winkler [3] foundation, Pasternak foundation, Hetenyi foundation and Vlasov foundation. Winkler [3] foundation is based on the assumption that the foundation's reaction $q(x, y)$ at any point $(x, y)$ on the plate is directly proportional to the deflection $w(x, y)$ at the point, and the proportionality constant, $k$, called the foundation modulus or Winkler modulus is a constant for the entire foundation. Thus, mathematically [10]

$$
q(x, y)=k w(x, y)
$$

Two parameter elastic foundation models

Filanenko Borodich, Hetenyi [5] and Pasternak [4] gave the expressions for the foundation reaction $q(x, y)$ in terms of two parameters as follows:

$$
q(x, y)=k w(x, y)-T \nabla^{2} w(x, y)
$$

where $k$ and $T$ are the foundation model parameters; and $\nabla^{2}$ is the Laplacian, for rectangular or circular foundations, and,

$$
q(x, y)=k w(x, y)-T \frac{d^{2} w(x, y)}{d x^{2}}
$$

For strip foundation

(for Filanenko Borodich foundation)

For Hekenyi [5] foundation,

$$
q(x, y)=k w(x, y)+D \nabla^{4} w(x, y)
$$

\footnotetext{
${ }^{*}$ Corresponding author
} 
where $D=\frac{E_{p} h_{p}^{3}}{12\left(1-\mu_{p}^{2}\right)}$

and $k$ and $D$ are the foundation model parameters.

$D=$ flexural rigidity of the elastic plate, $E_{p}$ and $\mu_{p}$ are the Young's modulus and Poisson's ratio of the plate material, $h_{p}$ is the plate thickness.

For Pasternak [4] foundation

$$
q(x, y)=k w(x, y)-G \nabla^{2} w(x, y)
$$

where $G=$ shear modulus of the shear layer, and $k$ and $D$ are the Pasternak foundation model parameters.

Mathematically, the two parameter elastic foundation models are equivalent. The only difference is the definition of the elastic foundation parameter, and the two parameter elastic foundation models are expressed as

$$
q(x, y)=k w(x, y)-k_{s} \nabla^{2} w(x, y)
$$

in which $k$ is the first elastic foundation parameter (Winkler modulus) and $k_{s}$ is the second elastic foundation parameter.

Two principal theories are used to describe plate structures: classical thin plate theory, also called Kirchhoff's plate theory and the shear deformable plate theory (Mindlin-Reissner's plate theory) used for moderately thick plates [11]. In this study, the Kirchhoff plate theory is adopted for the plate and the Winkler model adopted for the elastic foundation.

\section{Research aim and objectives}

The aim of this research is to apply the finite Fourier sine transform method to obtain solutions to the simply supported Kirchhoff plate on Winkler foundation. The specific objectives are:

(i) to transform the governing partial differential equation of equilibrium of Kirchhoff plate on Winkler foundation to an algebraic equation using the finite Fourier sine transformation.

(ii) to solve the resulting algebraic equation to obtain solution for any distribution of transverse load on the Kirchhoff plate on Winkler foundation problem.

(iii) to obtain solutions for particular types of transverse load - (a) point load $P_{0}$ applied at a known point ( $x_{0}$, $y_{0}$ ) on the plate domain; (b) uniformly distributed load of intensity $q_{0}$ over the entire plate domain.

\section{Methodology}

The finite Fourier sine transform method was introduced by Doetsch [12] as a technique for solving boundary value problems by integral transformation. Subsequently the method has been developed and generalised by other scholars and researchers such as Kneitz [13], Standhagen [14], Roettinger [15] and Brown [16]. The general philosophy of the integral transform methods, and in particular the finite Fourier sine transform method is that they simplify boundary value problems by eliminating partial derivatives with respect to one of the independent variables hence, the transformed equation has a reduced number of independent variables, and is easier to solve. Thus partial differential equations (PDEs) in terms of two independent variables are transformed to ordinary differential equations (ODEs) and ordinary differential equations are transformed to algebraic equations.

\section{Definitions}

The finite Fourier sine transform $s(u(x))$ of function $u(x)$ of the independent variable, $x$, is defined as:

$$
s_{n}=s(u(x))=\int_{0}^{l} u(x) \sin \frac{n \pi x}{l} d x
$$

where $n=1,2,3, \ldots$

$$
0 \leq x \leq l
$$

The finite Fourier sine transform of the derivatives are determined, using integration by parts, in terms of the finite Fourier sine transform of the unknown function $u(x)$. The double finite Fourier sine transform of a function $w(x, y)$ of two independent variable $x$ and $y$ defined over the rectangular domain $0 \leq x \leq a ; \quad 0 \leq y \leq b$ is given by

$$
\begin{aligned}
& w(m, n)=\int_{0}^{b} \int_{0}^{a} w(x, y) \sin \frac{m \pi x}{a} \sin \frac{n \pi y}{b} d x d y \\
& m=1,2,3, \ldots \quad n=1,2,3, \ldots
\end{aligned}
$$

where $w(m, n)$ is the double finite Fourier sine transform of $w(x, y)$. 
The inverse transform of $w(m, n)$ is

$$
w(x, y)=\frac{4}{a b} \sum_{m}^{\infty} \sum_{n}^{\infty} w(m, n) \sin \frac{m \pi x}{a} \sin \frac{n \pi y}{b}
$$

where $m=1,2,3,4, \ldots ; \quad n=1,2,3,4, \ldots$

The double finite Fourier sine transforms of partial derivatives and mixed partial derivatives of any function of two or more independent variables are obtained in terms of the transform of the unknown function by the use of integration by parts.

\section{Finite Fourier sine transform method for Rectangular Kirchhoff plate on Winkler foundation}

Consider a rectangular Kirchhoff plate $a \times b$ resting on a Winkler foundation as shown in Figure 1 .

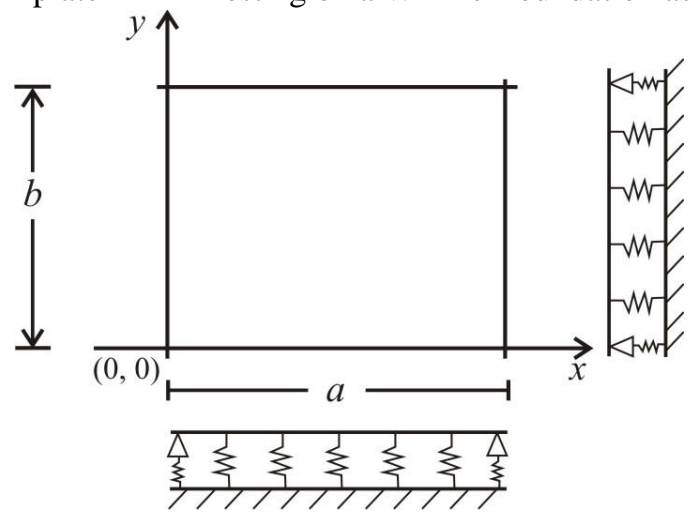

Figure 1: Rectangular Kirchhoff plate on Winkler foundation

The governing partial differential equation of equilibrium is given for homogeneous isotropic plates under static loads by:

$$
D \nabla^{4} w(x, y)+k w(x, y)=q(x, y)
$$

for $0 \leq x \leq a, \quad 0 \leq y \leq b$

where

$$
D=\frac{E h^{3}}{12\left(1-\mu^{2}\right)} \text { is the plate flexural rigidity, } \nabla^{4}=\frac{\partial^{4}}{\partial x^{4}}+2 \frac{\partial^{4}}{\partial x^{2} \partial y^{2}}+\frac{\partial^{4}}{\partial y^{4}}, k=\text { Winkler }
$$

modulus of subgrade reaction or Winkler constant, $w(x, y)$ is the deflection of the plate middle surface, $q(x, y)$ is the external distributed load applied to the plate, $x$ and $y$ are the inplane Cartesian coordinate variables describing the plate domain, $a$ and $b$ are the length and width of the plate or the inplane dimensions of the plate domain.

The governing equation can be expressed by

$$
\nabla^{4} w(x, y)+\frac{k w(x, y)}{D}=\frac{q(x, y)}{D}
$$

We seek to solve the problem for plates on Winkler foundations for the case of simply supported edges (at $x=0$, $x=a, y=0, y=b)$. The geometric and force boundary conditions at the simply supported edges are at $x=0, x=$ $a$,

$$
\begin{gathered}
w(x, y)=0 \\
\frac{\partial^{2} w}{\partial x^{2}}(x, y)=0 \\
\text { At } y=0, y=b \\
w(x, y)=0 \\
\frac{\partial^{2} w}{\partial y^{2}}(x, y)=0
\end{gathered}
$$

We apply the finite Fourier sine transform to both sides of Equation (11) to obtain

$$
\iint_{R^{2}}\left(\nabla^{4} w(x, y)+\frac{k}{D} w(x, y)\right) \sin \frac{m \pi x}{a} \sin \frac{n \pi y}{b} d x d y
$$




$$
=\iint_{R^{2}} \frac{q(x, y)}{D} \sin \frac{m \pi x}{a} \sin \frac{m \pi y}{b} d x d y
$$

where $R^{2}$ is given by:

$0 \leq x \leq a, 0 \leq y \leq b$

$$
\begin{gathered}
\int_{0}^{a} \int_{0}^{b} \nabla^{2} w(x, y) \sin \frac{m \pi x}{a} \sin \frac{m \pi y}{b} d x d y+\int_{0}^{a} \int_{0}^{b} \frac{k}{D} w \sin \frac{m \pi x}{a} \sin \frac{m \pi y}{b} d x d y \\
=\int_{0}^{a} \int_{0}^{b} \frac{q}{D} \sin \frac{m \pi x}{a} \sin \frac{m \pi y}{b} d x d y
\end{gathered}
$$

or

$$
\begin{aligned}
& \int_{0}^{a} \int_{0}^{b}\left(\frac{\partial^{4}}{\partial x^{4}}+2 \frac{\partial^{4}}{\partial x^{2} \partial y^{2}}+\frac{\partial^{4}}{\partial y^{4}}\right) w \sin \frac{m \pi x}{a} \sin \frac{n \pi y}{b} d x d y+\frac{k}{D} \int_{0}^{a} \int_{0}^{b} w \sin \frac{m \pi x}{a} \sin \frac{n \pi y}{b} d x d y \\
& =\frac{1}{D} \int_{0}^{a} \int_{0}^{b} q \sin \frac{m \pi x}{a} \sin \frac{n \pi y}{b} d x d y \\
& \int_{0}^{a} \int_{0}^{b}\left(\left(\frac{m \pi}{a}\right)^{4}+2\left(\frac{m \pi}{a}\right)^{2}\left(\frac{n \pi}{b}\right)^{2}+\left(\frac{n \pi}{b}\right)^{4}\right) w \sin \frac{m \pi x}{a} \sin \frac{n \pi y}{b} d x d y \\
& +\frac{k}{D} \int_{0}^{a} \int_{0}^{b} w \sin \frac{m \pi x}{a} \sin \frac{n \pi y}{b} d x d y=\frac{1}{D} \int_{0}^{a} \int_{0}^{b} q \sin \frac{m \pi x}{a} \sin \frac{n \pi y}{b} d x d y \\
& \left(\left(\frac{m \pi}{a}\right)^{2}+\left(\frac{n \pi}{b}\right)^{2}\right)^{2} \int_{0}^{a} \int_{0}^{b} w \sin \frac{m \pi x}{a} \sin \frac{n \pi y}{b} d x d y+\frac{k}{D} \int_{0}^{a} \int_{0}^{b} w \sin \frac{m \pi x}{a} \sin \frac{n \pi y}{b} d x d y \\
& =\frac{1}{D} \int_{0}^{a} \int_{0}^{b} q \sin \frac{m \pi x}{a} \sin \frac{n \pi y}{b} d x d y \\
& \left(\left(\frac{m \pi}{a}\right)^{2}+\left(\frac{n \pi}{b}\right)^{2}\right)^{2} w(m, n)+\frac{k}{D} w(m, n)=\frac{1}{D} q(m, n)
\end{aligned}
$$

where

$$
\begin{aligned}
& w(m, n)=\int_{0}^{a} \int_{0}^{b} w(x, y) \sin \frac{m \pi x}{a} \sin \frac{n \pi y}{b} d x d y \\
& q(m, n)=\int_{0}^{a} \int_{0}^{b} q(x, y) \sin \frac{m \pi x}{a} \sin \frac{n \pi y}{b} d x d y
\end{aligned}
$$

$w(m, n)$ is the double finite Fourier sine transform of the deflection $w(x, y)$ and $q(m, n)$ is the double finite Fourier sine transform of the distributed load $q(x, y)$.

Thus

$$
\left[\left(\left(\frac{m \pi}{a}\right)^{2}+\left(\frac{n \pi}{b}\right)^{2}\right)^{2}+\frac{k}{D}\right] w(m, n)=\frac{q(m, n)}{D}
$$

Solving for $w(m, n)$, we obtain 


$$
\begin{aligned}
& w(m, n)=\frac{q(m, n) / D}{\left(\left(\frac{m \pi}{a}\right)^{2}+\left(\frac{n \pi}{b}\right)^{2}\right)^{2}+\frac{k}{D}} \\
& w(m, n)=\frac{q(m, n) / D}{\left[\left(\left(\frac{m \pi}{a}\right)^{2}+\left(\frac{n \pi}{b}\right)^{2}\right)^{2}+\frac{k}{D}\right]} \\
& w(m, n)=\frac{q(m, n)}{D\left[\left(\left(\frac{m \pi}{a}\right)^{2}+\left(\frac{n \pi}{b}\right)^{2}\right)^{2}+\frac{k}{D}\right]}
\end{aligned}
$$

By inversion, the deflection is obtained as:

$$
\begin{gathered}
w(x, y)=\frac{4}{a b} \sum_{m}^{\infty} \sum_{n}^{\infty} w(m, n) \sin \frac{m \pi x}{a} \sin \frac{n \pi y}{b} \\
m=1,2,3, \ldots, \infty \quad n=1,2,3, \ldots, \infty \\
w(x, y)=\frac{4}{a b} \sum_{m}^{\infty} \sum_{n}^{\infty} \frac{q(m, n) \sin \frac{m \pi x}{a} \sin \frac{n \pi y}{b}}{D\left[\left(\left(\frac{m \pi}{a}\right)^{2}+\left(\frac{n \pi}{b}\right)^{2}\right)^{2}+\frac{k}{D}\right]}
\end{gathered}
$$

\section{Bending Moment Distributions}

The bending moment distributions $\left(M_{x x}\right.$ and $M_{y y}$ ) are obtained from the bending moment curvature relations i.e.

$$
\begin{aligned}
& M_{x x}=-D\left(\frac{\partial^{2} w}{\partial x^{2}}+\mu \frac{\partial^{2} w}{\partial y^{2}}\right) \\
& M_{y y}=-D\left(\frac{\partial^{2} w}{\partial y^{2}}+\mu \frac{\partial^{2} w}{\partial x^{2}}\right)
\end{aligned}
$$

By differentiation, and substitution of the derivatives, we obtain,

$$
\begin{aligned}
& \frac{\partial^{2} w}{\partial x^{2}}=\frac{4}{a b} \sum_{m}^{\infty} \sum_{n}^{\infty} \frac{-\left(\frac{m \pi}{a}\right)^{2} q(m, n) \sin \frac{m \pi x}{a} \sin \frac{n \pi y}{b}}{D\left[\left(\left(\frac{m \pi}{a}\right)^{2}+\left(\frac{n \pi}{b}\right)^{2}\right)^{2}+\frac{k}{D}\right]} \\
& \frac{\partial^{2} w}{\partial y^{2}}=\frac{4}{a b} \sum_{m}^{\infty} \sum_{n}^{\infty} \frac{-\left(\frac{n \pi}{b}\right)^{2} q(m, n) \sin \frac{m \pi x}{a} \sin \frac{n \pi y}{b}}{D\left[\left(\left(\frac{m \pi}{a}\right)^{2}+\left(\frac{n \pi}{b}\right)^{2}\right)^{2}+\frac{k}{D}\right]}
\end{aligned}
$$




$$
\begin{aligned}
& M_{x x}=-D \cdot \frac{-4}{a b} \sum_{m}^{\infty} \sum_{n}^{\infty} \frac{\left[\left(\frac{m \pi}{a}\right)^{2}+\mu\left(\frac{n \pi}{b}\right)^{2}\right] q(m, n) \sin \frac{m \pi x}{a} \sin \frac{n \pi y}{b}}{D\left[\left(\left(\frac{m \pi}{a}\right)^{2}+\left(\frac{n \pi}{b}\right)^{2}\right)^{2}+\frac{k}{D}\right]} \\
& M_{x x}=\frac{4 D}{a b} \sum_{m}^{\infty} \sum_{n}^{\infty} \frac{\left[\left(\frac{m \pi}{a}\right)^{2}+\mu\left(\frac{n \pi}{b}\right)^{2}\right] q(m, n) \sin \frac{m \pi x}{a} \sin \frac{n \pi y}{b}}{D\left[\left(\left(\frac{m \pi}{a}\right)^{2}+\left(\frac{n \pi}{b}\right)^{2}\right)^{2}+\frac{k}{D}\right]} \\
& M_{x x}=\frac{4}{a b} \sum_{m}^{\infty} \sum_{n}^{\infty} \frac{\left[\left(\frac{m \pi}{a}\right)^{2}+\mu\left(\frac{n \pi}{b}\right)^{2}\right] q(m, n) \sin \frac{m \pi x}{a} \sin \frac{n \pi y}{b}}{\left(\left(\frac{m \pi}{a}\right)^{2}+\left(\frac{n \pi}{b}\right)^{2}\right)^{2}+\frac{k}{D}} \\
& M_{y y}=\frac{4 D}{a b} \sum_{m}^{\infty} \sum_{n}^{\infty} \frac{\left[\left(\frac{n \pi}{b}\right)^{2}+\mu\left(\frac{m \pi}{a}\right)^{2}\right] q(m, n) \sin \frac{m \pi x}{a} \sin \frac{n \pi y}{b}}{D\left[\left(\left(\frac{m \pi}{a}\right)^{2}+\left(\frac{n \pi}{b}\right)^{2}\right)^{2}+\frac{k}{D}\right]} \\
& M_{y y}=\frac{4}{a b} \sum_{m}^{\infty} \sum_{n}^{\infty} \frac{\left[\left(\frac{n \pi}{b}\right)^{2}+\mu\left(\frac{m \pi}{a}\right)^{2}\right] q(m, n) \sin \frac{m \pi x}{a} \sin \frac{n \pi y}{b}}{\left(\left(\frac{m \pi}{a}\right)^{2}+\left(\frac{n \pi}{b}\right)^{2}\right)^{2}+\frac{k}{D}}
\end{aligned}
$$

Finite Fourier Sine transform solutions for point load $\boldsymbol{P}_{\mathbf{0}}$ at $x_{\mathbf{0}}, \boldsymbol{y}_{\mathbf{0}}$ where $0 \leq x_{0} \leq a, 0 \leq y_{0} \leq b$

For point load, $P_{0}$ applied at the point $\left(x_{0}, y_{0}\right)$ on the plate domain, the finite Fourier transform representation of the load, $q(m, n)$ is given by

$$
\begin{aligned}
& q(m, n)=\int_{0}^{a} \int_{0}^{b} q(x, y) \sin \frac{m \pi x}{a} \sin \frac{n \pi y}{b} d x d y \\
& q(m, n)=\int_{0}^{a} \int_{0}^{b} P_{0}\left(x-x_{0}\right) \delta\left(y-y_{0}\right) \sin \frac{m \pi x}{a} \sin \frac{n \pi y}{b} d x d y \\
& q(m, n)=P_{0} \sin \frac{m \pi x_{0}}{a} \sin \frac{n \pi y_{0}}{b}
\end{aligned}
$$

Then 


$$
w(m, n)=\frac{P_{0} \sin \frac{m \pi x_{0}}{a} \sin \frac{n \pi y_{0}}{b}}{D\left[\left(\left(\frac{m \pi}{a}\right)^{2}+\left(\frac{n \pi}{b}\right)^{2}\right)^{2}+\frac{k}{D}\right]}
$$

By inversion,

$$
\begin{aligned}
w(x, y)=\frac{4}{a b} \sum_{m}^{\infty} \sum_{n}^{\infty} \frac{P_{0} \sin \frac{m \pi x_{0}}{a} \sin \frac{n \pi y_{0}}{b} \sin \frac{m \pi x}{a} \sin \frac{n \pi y}{b}}{D\left[\left(\left(\frac{m \pi}{a}\right)^{2}+\left(\frac{n \pi}{b}\right)^{2}\right)^{2}+\frac{k}{D}\right]} \\
w(x, y)=\frac{4 P_{0}}{a b D} \sum_{m}^{\infty} \sum_{n}^{\infty} \frac{\sin \frac{m \pi x_{0}}{a} \sin \frac{n \pi y_{0}}{b} \sin \frac{m \pi x}{a} \sin \frac{n \pi y}{b}}{\left(\left(\frac{m \pi}{a}\right)^{2}+\left(\frac{n \pi}{b}\right)^{2}\right)^{2}+\frac{k}{D}} \\
M_{x x}=\frac{4}{a b} \sum_{m}^{\infty} \sum_{n}^{\infty} \frac{\left(\left(\frac{m \pi}{a}\right)^{2}+\mu\left(\frac{n \pi}{b}\right)^{2}\right) P_{0} \sin \frac{m \pi x_{0}}{a} \sin \frac{n \pi y_{0}}{b} \sin \frac{m \pi x}{a} \sin \frac{n \pi y}{b}}{\left(\left(\frac{m \pi}{a}\right)^{2}+\left(\frac{n \pi}{b}\right)^{2}\right)^{2}+\frac{k}{D}} \\
M_{y y}=\frac{4}{a b} \sum_{m}^{\infty} \sum_{n}^{\infty} \frac{\left(\left(\frac{n \pi}{b}\right)^{2}+\mu\left(\frac{m \pi}{a}\right)^{2}\right) P_{0} \sin \frac{m \pi x_{0}}{a} \sin \frac{n \pi y_{0}}{b} \sin \frac{m \pi x}{a} \sin \frac{n \pi y}{b}}{\left(\left(\frac{m \pi}{a}\right)^{2}+\left(\frac{n \pi}{b}\right)^{2}\right)^{2}+\frac{k}{D}} \\
M_{m}^{\infty} \sum_{n}^{\infty} \frac{\left(\left(\frac{m \pi}{a}\right)^{2}+\mu\left(\frac{n \pi}{b}\right)^{2}\right) \sin \frac{m \pi x_{0}}{a} \sin \frac{n \pi y_{0}}{b} \sin \frac{m \pi x}{a} \sin \frac{n \pi y}{b}}{\left(\left(\frac{m \pi}{b}\right)^{2}+\frac{k}{D}\right.}
\end{aligned}
$$

Finite Fourier Sine transform solutions for uniformly distributed load $q_{0}$ over the entire plate domain For uniformly distributed load of intensity, $q_{0}$ over the entire plate domain, the finite Fourier sine transform representation of the load $q(m, n)$ is given from Equation (21) by:

$$
q(m, n)=\int_{0}^{a} \int_{0}^{b} q_{0} \sin \frac{m \pi x}{a} \sin \frac{n \pi y}{b} d x d y
$$




$$
q(m, n)=\frac{4 q_{0} a b}{m n \pi^{2}}
$$

Then

$$
m=1,3,5, \ldots \quad n=1,3,5, \ldots
$$

$$
\begin{aligned}
& w(m, n)=\frac{\frac{4 q_{0} a b}{m n \pi^{2}}}{D\left[\left(\left(\frac{m \pi}{a}\right)^{2}+\left(\frac{n \pi}{b}\right)^{2}\right)^{2}+\frac{k}{D}\right]} \\
& m=1,3,5,7, \ldots \quad \frac{n=1,3,5,7, \ldots}{4 q_{0} a b} \sin \frac{m \pi x}{a} \sin \frac{n \pi y}{b} \\
& w(x, y)=\frac{4}{a b} \sum_{m}^{\infty} \sum_{n}^{\infty} \frac{m n \pi^{2}}{D\left[\left(\left(\frac{m \pi}{a}\right)^{2}+\left(\frac{n \pi}{b}\right)^{2}\right)^{2}+\frac{k}{D}\right]} \\
& w(x, y)=\frac{16 q_{0}}{\pi^{2}} \sum_{m}^{\infty} \sum_{n}^{\infty} \frac{\sin \frac{m \pi x}{a} \sin \frac{n \pi y}{b}}{m n D\left[\left(\left(\frac{m \pi}{a}\right)^{2}+\left(\frac{n \pi}{b}\right)^{2}\right)^{2}+\frac{k}{D}\right]}
\end{aligned}
$$

From the moment-displacement relations, the bending moments are given by:

$$
\begin{aligned}
& M_{x x}=\frac{16 q_{0}}{\pi^{2}} \sum_{m}^{\infty} \sum_{n}^{\infty} \frac{\left(\left(\frac{m \pi}{a}\right)^{2}+\mu\left(\frac{n \pi}{b}\right)^{2}\right) \sin \frac{m \pi x}{a} \sin \frac{n \pi y}{b}}{m n\left[\left(\left(\frac{m \pi}{a}\right)^{2}+\left(\frac{n \pi}{b}\right)^{2}\right)^{2}+\frac{k}{D}\right]} \\
& M_{y y}=\frac{16 q_{0}}{\pi^{2}} \sum_{m}^{\infty} \sum_{n}^{\infty} \frac{\left(\left(\frac{n \pi}{b}\right)^{2}+\mu\left(\frac{m \pi}{a}\right)^{2}\right) \sin \frac{m \pi x}{a} \sin \frac{n \pi y}{b}}{m n\left[\left(\left(\frac{m \pi}{a}\right)^{2}+\left(\frac{n \pi}{b}\right)^{2}\right)^{2}+\frac{k}{D}\right]}
\end{aligned}
$$

\section{Discussion of Results}

The finite Fourier sine transform method has been successfully applied to the solution of the fourth order partial differential equation governing the Kirchhoff plate on Winkler foundation problem when the four edges $x=0, x=a, y=0, y=b$ are simply supported, and the plate domain carries transverse distributed load. The double finite Fourier sine transformation was applied to both sides of the governing partial differential equation (PDE) which become transformed to an algebraic equation in the transform space $(m, n)$ variables given by Equation (22); where $w(m, n)$ is the unknown deflection in terms of the transform variables $(m, n)$. The solution for deflection in the finite Fourier sine transform variables was obtained as Equations (23), or (24) or (25). The solution for deflection is the plate domain space variables was obtained by inversion of Equations (23) or (24) or (25) and given as Equation (27); for the general problem of any distributed transverse load. Bending moment expressions were obtained by using the moment-displacement relations as Equations (34) and (35). The general results obtained were then used to obtain solutions for two particular cases of transverse load. For a point load $P_{0}$ applied at the point $\left(x_{0}, y_{0}\right)$ the deflections were obtained in terms of the transform space variables as Equation (39) and in terms of the plate domain space variables as Equation (41). Bending moment expressions were obtained for the point load case as Equations (44) and (45). For the case of uniformly 
distributed load of intensity $q_{0}$ over the entire plate domain, the deflection was obtained in terms of the transform space variables as Equation (48), and in terms of the plate domain variables as Equation (50). Bending moment expressions were obtained as Equations (51) and (52) for uniform transverse loads. The expressions obtained for both the deflection in the plate domain and the bending moments in the plate domain for the two particular cases of transverse load were identical with the expressions obtained using the Navier double Fourier series method for the Kirchhoff plate on Winkler foundation problem; as presented in Ugural [17].

The finite Fourier sine transform solutions obtained for deflection for the case of point load applied at the point $\left(x_{0}, y_{0}\right)$ and for uniformly distributed load applied over the entire plate domain were the exact solutions for the Kirchhoff plate on Winkler foundation problem; being identical solutions with the Navier series solutions that yielded exact results. Similarly, bending moment expressions obtained were the exact bending moment distributions on the plate.

\section{Conclusions}

The following conclusions can be made from this study:

(i) the double finite Fourier sine transform method effectively transforms the Kirchhoff plate on Winkler foundation governing PDE to an algebraic equation in terms of the transform variables $(m, n)$.

(ii) the double Fourier sine transformation effectively transforms the boundary value problem to an algebraic problem.

(iii) the double Fourier sine transform method gives exact solutions to the problem of homogeneous isotropic Kirchhoff plate on Winkler foundation for the case of: (a) general distribution of transverse loads, (b) point load applied at a point on the plate domain, and (c) uniformly distributed load over the entire plate region.

\section{References}

[1] Timoshenko S. and Woinowsky-Krieger S.: Theory of Plates and Shells, Second Edition, McGraw Hill Book Company, Tokyo 580pp., 1959.

[2] Ventsel E. and Krauthammer T.: Thin Plates and Shells: Theory, Analysis and Applications. Marcel Dekker Inc USA, 666pp., 2001.

[3] Winkler E. Die Lehre vonder elastizitat und festigkeit, Prague, 1867.

[4] Pasternak P.L.: On a new method of analysis of an elastic foundation by means of two foundation constants (in Russian) Gosud.Izd.Lit po Stroitelstvu, Arkhitekture, Moscow, 1954.

[5] Hetenyi M.: Beams on Elastic Foundation. The University of Michigan Press, Ann Arbor, Michigan, 1946

[6] Gorbunov-Posadov, M.L.: On ways of development of the theory of structures on elastic foundation (in Russian), Osnovania Fundamenty; Mekhanika Gruntov, no1, p.1. 1963.

[7] Albert P. and Kovacs M.: Modelling of Reinforced Soil, Periodica Polytechnica Ser. Civ. Eng. Vol. 47, No 2, pp. 169-174. 2003.

[8] Caselunghe A. and Eriksson J.: Structural Element Approaches for Soil Structure Interaction. MSc Thesis, Chalmer University of Technology Göteborg Sweden. 2012

[9] Ghaitani, M.M., Esmaeili, H.A. and Kolahchi R.: Elastic foundation effect on bending behaviour of oil pipelines. International Conference on Civil, Biological and Environmental Engineering (CBEE), May 27-28, 2014, Istanbul, Turkey.

[10] Rajpurohit V.K., Gore, N.G. and Sayagavi V.G.: Analysis of Structures Supported on Elastic Foundation. International Journal of Engineering and Advanced Technology (IJEAT), Volume 4, Issue 1, October 2014

[11] Wen P.H.: The fundamental solution of Mindlin plates resting on an elastic foundation in the Laplace domain and its applications. International Journal of Solids and Structures, 45 (2008), pp 1032-1050, Elservier. 2008

[12] Doetsch, G.: Integration von Differentialgleichungen vermittels der endlichen Fourier transformation Math, Ann, 112, pp 52-68. 1935.

[13] Kneitz K.: Lösung von Randwert problem bei systemem gewöhullicher Differentialgleichungen vermittels der endlichen Fourier transformation Math. Zeit, 44, pp 266-291. 1938.

[14] Strandhagen G.: Use of sine transformation for non-simply supported beams Quart. Appl. Math 1, pp. 346-348. 1944.

[15] Roettinger, I.: An operational approach to the solution of boundary value problems by generalised Fourier series. Bull. American Math. Soc., 51, p 67. 1945.

[16] Brown H.K.: Resolution of temperature problems by use of finite Fourier transformations Bull. American Math. Soc., 50, pp. 376385. 1944

[17] Ugural A.C.: Stresses in Plates and Shells, Second Edition. McGraw Hill Toronto, 502pp. 1999. 